\title{
Role of Artificial Intelligence within the Telehealth Domain
}

\section{Official 2019 Yearbook Contribution by the members of IMIA Telehealth Working Group}

\author{
Craig Kuziemsky ${ }^{1}$, Anthony J. Maeder ${ }^{2}$, Oommen John ${ }^{3}$, Shashi B. Gogia ${ }^{4}$, Arindam Basu ${ }^{5}$, \\ Sushil Meher ${ }^{6}$, Marcia Ito ${ }^{7}$ \\ 1 Telfer School of Management, University of Ottawa, Ottawa, Canada \\ ${ }^{2}$ College of Nursing \& Health Sciences, Flinders University, Adelaide, Australia \\ ${ }^{3}$ George Institute for Global Health, University of New South Wales, New Delhi, India \\ 4 Society for Administration of Telemedicine and Healthcare Informatics, New Delhi, India \\ 5 University of Canterbury School of Health Sciences, Christchurch, New Zealand \\ ${ }^{6}$ All India Institute of Medical Sciences, New Delhi, India \\ 7 IBM Research, Brazil
}

\begin{abstract}
Summary
Objectives: This paper provides a discussion about the potential scope of applicability of Artificial Intelligence methods within the telehealth domain. These methods are focussed on clinical needs and provide some insight to current directions, based on reports of recent advances.

Methods: Examples of telehealth innovations involving Arrificial Intelligence to support or supplement remote health care delivery were identified from recent literature by the authors, on the basis of expert knowledge. Observations from the examples were synthesized to yield an overview of contemporary directions for the perceived role of Artificial Intelligence in telehealth.

Results: Two major focus areas for related contemporary directions were established. These were first, quality improvement for existing clinical practice and service delivery, and second, the development and support of new models of care. Case studies from each focus area have been chosen for illustration purposes. Conclusion: Examples of the role of Artificial Intelligence in delivery of health care remotely include use of tele-assessment, tele-diagnosis, tele-interactions, and tele-monitoring. Further developments of underlying algorithms and validation of methods will be required for wider adoption. Certain key social and ethical considerations also need consideration more generally in the health system, as Artificial-Intelligence-enabled-telehealth becomes more commonplace.
\end{abstract}

\section{Keywords}

Telemedicine; Arrificial Intelligence; quality of health care; delivery of health care

Yearb Med Inform 2019:35-40

http://dx.doi.org/10.1055/s-0039-1677897

\section{Introduction}

The IMIA Telehealth Working Group consists of over 30 members worldwide, comprising clinicians and telehealth experts working as academics, data scientists, entrepreneurs, promoters, and researchers. This invited article outlines the potential scope of applicability of Artificial Intelligence (AI) methods within the telehealth domain.

We consider the importance, current, and possible future uses of $\mathrm{AI}$, and then outline the potential opportunities for addressing some of the system level challenges in implementation. We start by discussing the overall role of AI in telehealth after which we provide some specific scenarios wherein $\mathrm{AI}$ can improve health outcomes and enhance stakeholder experience and acceptance. We conclude with some comments on social and ethical considerations.

\section{Telehealth Paradigm in Health Care Delivery}

Telehealth uses information and communication technologies to transfer medical information for the delivery of clinical and educational services [1]. It attempts to over- come challenges of health service delivery due to time, distance, and difficult terrains, enabling cost-effectiveness and better access in both developed and developing world settings. It has a special role during emergencies like earthquakes and floods [2]. Additionally, longer lifespan and associated rising incidence of chronic diseases have increased care demand and complexity, necessitating longer interactions between patients and providers (as well as among providers), and thereby increasing the need for tele-healthcare support [3].

Telehealth has been classified historically as synchronous using real-time electronic communication, or asynchronous using store-and-forward communication. Recently a third form has been recognised as remote (tele) monitoring, involving data collection through distributed devices including Internet of Things (IOT). The last World Health Organisation global eHealth observatory survey [4] noted four exemplary well-embedded telehealth services: tele-radiology, tele-pathology, tele-dermatology and tele-psychiatry; of these, the first three follow asynchronous models of care and the fourth is synchronous. This demonstrates an inherent difficulty in replacing or augmenting real-time clinician-delivered services. Furthermore, the same survey revealed that over $60 \%$ of 
respondents identified lack of information on telehealth-related clinical practice to be a great impediment to wider uptake.

\section{Artificial Intelligence in Telehealth}

A recent analysis of contemporary trends in telehealth [5] proposed that two dominant drivers for change were emerging: i) high volume demand, due to the increasing difficulty to physically co-locate the patient, the clinician(s), and the associated data, and ii) high criticality applications, where specialised expertise is necessary at the precise moment of clinical demand. Irrespective of the modality used, actual delivery of care inevitably necessitates some face-to-face clinical interaction, with the required periodicity depending on the circumstances. In telehealth, Information and Communication Technology (ICT) tools can be used to address the issues of misdistribution of the demand versus supply of healthcare services. AI could assist with this issue by developing algorithms to match the availability of care providers with appropriate clinical skills to the need for such skillsets in the immediate vicinity. However, telehealth introduces several operational issues, such as when the telecommunication link fails, or when the remote care clinician is not available remotely. AI could potentially alleviate such situations by providing mechanisms for human or virtual interactions to occur, and thereby address difficulties in timing and availability of clinicians (such as the time taken to understand the patient's problem or taking a history).

Increasing innovation and discovery is making care provision more complex, beyond the scope of any single clinical provider. AI can support the development of knowledge of clinical processes: for example, how to leverage new innovations in therapies and procedures through personalisation to match patient cohorts or individual profiles. Increasing longevity and chronic disease management in multi-morbidity situations needs a multi-disciplinary team approach with continued care being provided within the community, ideally at home.
When coordinated care and continuum of care pathways are not followed, health care equality as well as quality suffer. There is a need for seamless communications and connections to occur across different elements of health care delivery: not all team members can always be present, which creates a need for tele-support or remote care. AI again could help address this need, both by enabling the intelligent information and communication environment in which clinicians could interact as well as maintain a detailed virtual knowledge base for the progression of the patient's condition and his/her management.

Russell and Norvig [6] in 1995 described the field of artificial intelligence comprehensively as including "problem solving and search, logic and inference, planning, probabilistic reasoning and decision making, learning, communication, perception, and robotics". They further contended that computers, in the domain of artificial intelligence, could serve as "intelligent agents" and would mimic, if not match, humans in thought processes and reasoning to the extent that computers might emulate human cognitive behaviour and mimic human cognitive performance. Considering this wide range of possibilities, Pacis et.al. [7] recently summarised the potential impact of AI in telehealth around four emergent trends based on distinctive health care purposes: patient monitoring, healthcare information technology, intelligent assistance and diagnosis, and information analysis collaboration. We argue that they can be merged to two main areas of focus and become topics which we discuss in the rest of this paper: firstly, quality improvement for existing clinical practice and service delivery, and secondly, the development and support of new models of care.

\section{Telehealth, Al, and Quality Improvement}

In recent years, there has been a rapid and exponential increase in the quantum of health-related digital data that is generated by the citizens themselves as well as healthcare providers. There has consequently been a movement towards universal electronic health record systems, and automated aggregation of patient information through proliferation of healthcare information technology. The availability of large data sets combined with the rapid evolution of computational data science (including AI-based machine learning methods) offer promising opportunities for extracting new inferences and actionable insights that have a potential to improve health outcomes significantly. This more sophisticated data-enriched environment in turn allows better clinical decision-making through support by automated means, encouraging moves towards intelligent assistance and diagnosis.

\section{Example: Clinical Assessment and Evaluation}

Before routine use of high-end diagnostic tools and imaging devices like MRI and CT became common, clinical evaluation largely depended on a simple personal history and examination. Peterson et al. in 1992 [8] reported a $76 \%$ contribution by history and $11 \%$ by examination for an initial diagnosis. Roshan and Rao in 2000 [9] found the role of history to be roughly the same but the role of examination to be even less (7.6\%). As of today, the art of history taking and examination is getting compromised and health care is suffering as a result.

Investigations make telecare easier as such data is easy to collect and transfer. The contribution of high-end imaging Ultrasound (gallstones, liver abscess), CT (tumor in frontal area), MRI, etc., towards making a conclusive diagnosis has downgraded the importance of history taking and sometimes even obviates the need for a cursory clinical examination. The quality increase in healthcare delivery from this added sophistication has not been commensurate with the costs incurred. History taking is painstaking and consumes much of a physician's time, so it tends not to be used to the extent feasible via telemedicine. This is regrettable, as that is a component which is easily possible remotely and requires no special equipment. High end investigations, on the other hand, require much more remote infrastructure making it costly and decreasing the cost benefit advantage of telecare. 
For an experienced clinician, many clues pointing to a person's problem lie in the history. Not only does it guide the diagnosis, but it also leads to a more focussed investigation approach. AI can make history taking easier by providing prompts to the process and clues to the diagnosis, and also asking the right next set of questions based on the answer saving the clinician time. For example, a person suffering long-term with chronic, dull aching pain in the upper abdomen without interference with sleep is likely suffering from gastritis. A summary of such questions being asked in sequence can be delivered as part of a telehealth application, and may be implemented efficiently using mobile ICT.

Such systems can even support decision making when it is impossible to contact a clinician e.g., chest pain with likelihood of myocardial infarction means an urgent consideration for the use of streptokinase or at least Sorbitrate or Aspirin to be administered remotely. Benefits from such questions and conclusions thereof can be availed of directly by the patient with the assistance of a local nurse practitioner. Such questioning can be assisted by easy to use symbols within the user interface which all can understand.

\section{Example: Tele-diagnosis of Clinical Conditions}

Medical diagnosis has moved from clinical-examination-based in earlier days, to largely evidence-based supported by the doctor's own interpretation based on his/ her experience and skill. It is here that AI in last few years has begun to play a highly supportive role to doctors when analyzing the evidence. This has special relevance in oncology [10]: considering the progress of diseases that could relate to the formation of cancers, different disease diagnosis patterns might reflect different chances of cancer risks. Modeling disease progression and variants in disease trajectories helps in prediction [11]. By applying machine learning methods to large datasets of disease populations, $\mathrm{AI}$ is on its way globally to making a significant impact on the way doctors could diagnose a potential disease.
A well-known existing area for tele-diagnosis is tele-dermatology, which lends itself well to automation through AI. Currently, diagnostic accuracy for melanoma is dependent on the experience and training of the treating doctor. A recent study has shown that a computer algorithm using convolutional neural networks outperformed the majority of 58 dermatologists tested in accurately diagnosing melanoma, with a median area under the receiver operating characteristic curve of 0.86 compared with $0.79, \mathrm{P}<0.01$ [12]. Another study demonstrated classification of skin lesions using a deep convolutional neural networks $(\mathrm{CNN})$, trained end-to-end from images directly, using only pixels and disease labels as inputs. The CNN achieves performance on par with all tested experts across tasks of identification of the most common cancers, and identification of malignant melanoma the deadliest skin cancer, demonstrating an artificial intelligence capable of classifying skin cancer with a level of competence comparable to dermatologists [13]. Similar promise is offered in other areas of automated diagnosis such as breast cancer or cervical cancer screening.

\section{Telehealth, Al, and New Models of Care}

Globally there has been a significant increase in burden of chronic diseases. This combined with a growing base of aging populations with multiple morbidities, and the existing models of healthcare delivery are overstressed and unsustainable. Telehealth offers promising alternatives to utilize ICT optimally for remote healthcare diagnosis, monitoring, and delivery of care. Nevertheless, a number of system level challenges have restrained transformative telehealth models that have been scaled up at national or regional levels to exploit the full potential of delivering healthcare over distance. A recent review of telehealth interventions concluded that the implementation of a complex innovation such as remote care requires it to organically evolve, be responsive and adaptable to the local health and social care system and driven by support from front-line staff and management [14]. The increased capabilities for health care offered by patient monitoring must therefore be exercised in an environment of information analysis and collaboration by care team members and health systems agencies.

\section{Example: Conversational Agents and Virtual Assistants}

A natural progression from human-to-human interaction in telehealth is the development of computer-based generation and understanding of conversation to enable computer-to-human interaction. Online therapeutic and health counseling interventions have long acknowledged the utility of the technology that allows for variations between human-guided, patient-guided, and computer-guided approaches [15]. The value of synchronous and task-oriented computer-generated dialogue has been observed for mental health applications [16] and a broader range of applications has been noted in recent years [17]. Automated conversational interactions offer many other opportunities across the care spectrum to augment and in some cases replace human carer tasks. These may include:

- reminders and motivational messages e.g., for medication, nutrition, and exercise;

- routine condition checks and health maintenance, based on personal monitoring data;

- answering of health queries and provision of targeted health information and education;

- providing a personalised means to address social isolation and community involvement;

- acting as an intermediary or broker entity between multiple carers or service agencies.

The nature and complexity of conversational agent (or virtual assistant) solutions can vary considerably. For simple tasks, which are little more than a message or cue for informing the recipient, an audio voice or visual text communication is often adequate, and can also convey a minimal complexity response such as a confirmatory acknowledgement. Speech-text conversion utilities and chatbots capable of audio or typed inputs and outputs are examples of such technologies. They 
may have highly constrained conversational models embedded in them, capable of recognising and constructing only a limited range of phrases for a prespecified purpose. These solutions are better suited for interactions where the context of the situation and the user are simple and clearly established. AI mechanisms for these agents are typically rule-based using expert systems or decision tree logical constructs.

Schumaker et al. [18] characterise a chatbot as a system that "seeks to mimic conversation rather than understanding it". Examples of chatbots include responding to sales enquiries, travel directions, or performing search engine tasks. These have been widely adopted by the business sector as online assistants or first response agents. The first chatbot called ELIZA [19] was able to establish a conversation with human beings and mimic their conversational models. It was based on the rephrasing of input sentences, when these matched a set of pre-defined rules. Another evolving technology is voice recognition e.g. Cortana (MS Windows $\left.{ }^{\circledR}\right)$, Alexa $\left(\right.$ Amazon $\left.{ }^{\circledR}\right)$, Siri $\left(\operatorname{Mac}{ }^{\circledR}\right)$, Google Home ${ }^{\circledR}$ ...etc. [20,21]. Such technologies make it possible to have a service that can attend to a patient and caregiver in need of help at any time. Hybrid technologies where both humans and chatbots interact with patients and caregivers can also exist.

Health applications where virtual assistants can provide a viable supplementation or alternative to traditional healthcare delivery models include dealing with cognitively impaired individuals [22], improving accessibility of online clinical information [23], or providing avatar-based patient agents for the elderly [24]. Such cases rely upon a more sophisticated conversational purpose and knowledge base, and the level of AI complexity rises with a deeper understanding by the AI agent through data accumulation. It may be necessary to incorporate aspects of affective behaviour, using multimodal contextual awareness mechanisms to enable an authentic conversational dialogue [25]. For example, if issues arising from a patient's past interactions or past medical history need to be considered in making conversational decisions, a personalised model of the individual's context will be needed in addition to the context model for the current conversation.

\section{Example: Remote Patient Monitoring and Management}

Remote monitoring (or tele-monitoring) involves data acquisition using an appropriate sensor, transmission of data from patient to clinician, integration of data with other data describing the state of the patient (e.g. sourced from the electronic health record), synthesis of an appropriate action or response or escalation in the care of the patient with associated decision support, and storage of data [26]. AI systems for tele-monitoring depend on and also expand the scope of other health system ICT components. They can potentially outperform humans in many ways. They consistently execute their instructions in a mathematical fashion, with a fundamental reliance on inbuilt logic moderated by statistical evidence extracted by machine learning methods from large-scale datasets. They can immediately incorporate and co-ordinate data from additional tools like location finders (GPS), accelerometers, motion sensors, gyroscopes, ... etc. Sourcing such additional data by humans is tedious and would need education and training for incorporation in care delivery.

Tele-monitoring has been evaluated for the distant surveillance of patients with chronic disease, such as chronic heart failure [27], chronic obstructive pulmonary disease (COPD) [28], and diabetes mellitus [29]. In COPD, AI methods have been applied to the management and surveillance of the condition. A Classification and Regression Tree (CART) algorithm for the early identification of patients at high risk of an imminent exacerbation has been validated using telehealth measurement data recorded from patients with moderate/severe COPD living at home [30]. Similar approaches could be used as a real-time exacerbation event detector in a number of chronic conditions.

Apart from these above deterioration-oriented approaches, management of recovery situations provide another patient monitoring opportunity for telehealth. For example, software to measure wound size [31] can supplement visual assessment to give improvement in performance and assist remote care. In the example case, a special computer readable scale is provided, as well as applying automated image processing methods for changing the contrast of the image to help the sizing and automatic calculation of boundary and area. Manual oversight and correction are still deemed to be needed and may continue to be a necessary option for much AI-based tele-monitoring at least in the near future.

\section{Other Considerations and Issues}

While AI offers much potential for enhancing care delivery through telehealth tools, it is also important that we consider the social and ethical aspects of using it. AI, like other technological advances in healthcare, will cause disruption to many aspects of healthcare delivery including workflows, communication, access to services, and the engagement between different providers and patients [32]. Implementation is always the "last-mile" problem and our focus needs not to be on developing new AI tools or algorithms but rather on developing approaches for embedding $\mathrm{AI}$ in society.

The medical informatics community has long been studying the occurrence of unintended consequences (UICs) post HIT implementation. These UICs occur, not necessarily out of negligence on the part of system designers, but rather because of our inability to account for the new types of connectivity and communication practices that arise post-HIT implementation [33-35].

Like all new advancements, AI-enabled telehealth will go through a crest of excitement and potential, followed by a trough of disappointment and fear before reaching stability. Our efforts should be to achieve stability early. We offer four key social and ethical considerations for enabling AI-enabled telehealth.

1. Ensuring equity - While AI and technology can enhance access to and delivery of services, it can also increase the divide between the have and have nots [36]. We must ensure that technology-enabled healthcare delivery enables service delivery for those who need it most, such as people in rural and remote areas as well as in under-developed countries. 
2. Monitoring the technological divide - The world's population is diverse. Some populations are proficient users of technology while others are not. Persons expected to benefit most from advances in telehealth like the aged and extremely ill may be limited in their use of technology. We must ensure that AI applications do not increase the digital divide, but rather enhance our ability to provide quality patient-centered care delivery to all citizens [37].

3. AI is only a means - HIT implementation in healthcare has been described as a journey, not a destination [38], and as the digitization of healthcare proceeds at record speeds this becomes more apparent than ever. We must embrace the notion of AI to be part of a learning health care system [39] with a pragmatic approach with regards to the design and delivery of AI-enhanced tools.

4. People must come first - Healthcare is first and foremost about people. AI tools will change how interactions occur between different health system agents and we need to ensure that the focus of these tools are outcomes such as the empowerment of patients and a reduction in provider burnout [40].

\section{Conclusions}

The above discussion suggests that AI-enabled telehealth offers contributions in the form of quality improvement and enhancement of existing practice, as well as the instigation of new models of care. Examples provided of the role of $\mathrm{AI}$ in remote delivery of health care include the use of tele-assessment, tele-diagnosis, tele-interactions and tele-monitoring. Further development of underlying algorithms and validation of methods will be required for wider adoption.

Certain key social and ethical considerations also need to be taken into account as AI-enabled telehealth becomes more commonplace. Unlike humans, AI systems never lack motivation or drive, but without emotions there is an inability to assess moral good or bad, with little understanding of the consequences. Most AI methods require a substantial learning phase which can only achieve reliability after a very long time, and hence should be subject to continuous testing and refinement to better develop the human-AI interchange. Within the telehealth domain, control is even more difficult as some of the service delivery components may not have a human immediately in the loop. In such cases if things do go wrong, the question of who should be targeted for redress will be a challenge for society.

\section{References}

1. World Health Organisation. A health telematics policy in support of WHO's Health-For-All strategy for global health development: report of the WHO group consultation on health telematics, 11-16 December, Geneva, 1997. Geneva: World Health Organization; 1998.

2. Ajami S, Lamoochi P. Use of telemedicine in disaster and remote places. J Educ Health Promot 2014;3:26

3. McLean S, Protti D, Sheikh A. Telehealthcare for long term conditions. BMJ $2011 \mathrm{Feb}$ 3;342:d120.

4. World Health Organization. Telemedicine: opportunities and developments in member states. Report on the second global survey on eHealth. Geneva: World Health Organization; 2010.

5. Wilson LS, Maeder AJ. Recent directions in telemedicine: review of trends in research and practice. Healthc Inform Res 2015 Oct 1;21(4):213-22.

6. Russell S, Norvig P. Artificial intelligence - a modern approach. Prentice-Hall; 1995.

7. Pacis DM, Subido Jr ED, Bugtai NT. Trends in telemedicine utilizing artificial intelligence. In: AIP Conference Proceedings 2018 Feb 13;1933(1):040009.

8. Peterson,MC, Holbrook JH, Von Hales D, Smith NL, Staker LV. Contributions of the history, physical examination, and laboratory investigation in making medical diagnoses. West J Med 1992 Feb;156(2):163-5.

9. Roshan M, Rao A. A study on relative contributions of the history, physical examination and investigations in making medical diagnosis. J Assoc Physicians India $2000 ; 48(8): 771-5$.

10. Liang CW, et al. Using Machine Learning to Predict Cancer Risk by Clinical Diagnosis History - A Case Study of Liver Cancer. In preparation.

11. Haenssle HA, Fink C, Schneiderbauer R, Toberer F, Buhl T, Blum A, et al. Man against machine: diagnostic performance of a deep learning convolutional neural network for dermoscopic melanoma recognition in comparison to 58 dermatologists. Ann Oncol 2018 May 28;29(8):1836-42.

12. Esteva A, Kuprel B, Novoa RA, Ko J, Swetter SM, Blau HMet al. Dermatologist-level classification of skin cancer with deep neural networks. Nature $2017 \mathrm{Feb} ; 542$ (7639):115.
13. Goldstein BA, Navar AM, Pencina MJ, Ioannidis JP. Opportunities and challenges in developing risk prediction models with electronic health records data: a systematic review. J Am Med Inform Assoc 2017;24(1):198-208.

14. Hendy J, Chrysanthaki T, Barlow J, Knapp M, Rogers A, Sanders C, et al. An organisational analysis of the implementation of telecare and telehealth: the whole systems demonstrator. BMC Health Serv Res 2012 Dec;12(1):403.

15. Bara, A, Klein B, Proudfoot JG. Defining internet-supported therapeutic interventions. Ann Behav Med 2009 Aug 1;38(1):4-17.

16. Hoermann S, McCabe KL, Milne DN, Calvo RA. Application of synchronous text-based dialogue systems in mental health interventions: systematic review. J Med Internet Res 2017 Aug;19(8).

17. Laranjo L, Dunn AG, Tong HL, Kocaballi AB, Chen J, Bashir R, et al. Conversational agents in healthcare: a systematic review. J Am Med Inform Assoc 2018 Jul 11;25(9):1248-58.

18. Schumaker RP, Ginsburg M, Chen H, Liu Y. An evaluation of the chat and knowledge delivery components of a low-level dialog system: The az-alice experiment. Decis Support Syst 2007 January;42:2236-46.

19. Weizenbaum J. ELIZA - A computer program for the study of natural language communication between man and machine. Communications of the ACM 1966;9(1):36-45.

20. Klopfenstein LC, Delpriori S, Malatini S, Bogliolo A. The rise of bots: a survey of conversational interfaces, patterns, and paradigms. In: Proceedings of the 2017 Conference on Designing Interactive Systems 2017 Jun 10:555-65.

21. Lisetti C, Amini R, Yasavur U. Now all together: overview of virtual health assistants emulating face-to-face health interview experience. KI-Künstliche Intelligenz 2015 Jun 1;29(2):161-72.

22. Yaghoubzadeh R, Kramer M, Pitsch K, Kopp S. Virtual agents as daily assistants for elderly or cognitively impaired people. In: International Workshop on Intelligent Virtual Agents 2013 Aug 29. Berlin, Heidelberg: Springer; 2013. p. 79-91.

23. Bickmore TW, Utami D, Matsuyama R, Paasche-Orlow MK. Improving access to online health information with conversational agents: a randomized controlled experiment. J Med Internet Res 2016 Jan; 18(1).

24. Shaked NA. Avatars and virtual agents-relationship interfaces for the elderly. Healthc Technol Lett 2017 Jun 28:4(3):83.

25. Riccardi G. Towards healthcare personal agents. In: Proceedings of the 2014 Workshop on Roadmapping the Future of Multimodal Interaction Research including Business Opportunities and Challenges 2014 Nov 16. ACM; 2014. p. 53-6.

26. Nangalia V, Prytherch DR, Smith GB. Health technology assessment review: Remote monitoring of vital signs-current status and future challenges. Crit Care 2010 Oct;14(5):233.

27. Inglis SC, Clark RA, McAlister FA, Stewart S, Cleland JG. Which components of heart failure programmes are effective? A systematic review and meta-analysis of the outcomes of structured telephone support or telemonitoring as the primary component of chronic heart failure management in 8323 patients: abridged Cochrane Review. Eur 
J Heart Fail 2011 Sep;13(9):1028-40.

28. Bolton CE, Waters CS, Peirce S, Elwyn G. Insufficient evidence of benefit: a systematic review of home telemonitoring for COPD. J Eval Clin Pract 2011 Dec;17(6):1216-22.

29. Polisena J, Tran K, Cimon K, Hutton B, McGill S, Palmer K. Home telehealth for diabetes management: a systematic review and meta-analysis. Diabetes Obes Metab 2009 Oct;11(10):913-30.

30. Mohktar MS, Redmond SJ, Antoniades NC, Rochford PD, Pretto JJ, Basilakis J, et al. Predicting the risk of exacerbation in patients with chronic obstructive pulmonary disease using home telehealth measurement data. Artif Intell Med 2015 Jan 1;63(1):51-9.

31. Thawer HA, Houghton PE, Woodbury MG, Keast D, Campbell K. Computer-assisted and manual wound size measurement. Ostomy Wound Manage 2002;48(10):46-53.

32. Loh E. Medicine and the rise of the robots: a qualitative review of recent advances of artificial intelligence in health. BMJ Leader 2018;2:59-63.
33. Coiera E, Ash J, Berg M. The Unintended Consequences of Health Information Technology Revisited. Yearb Med Inform 2016:163-9.

34. Kuziemsky CE, Randell R, Borycki EM. Understanding Unintended Consequences and Health Information Technology: Contribution from the IMIA Organizational and Social Issues Working Group. Yearb Med Inform 2016(1):53-60.

35. Gogia SB, Maeder A, Mars M, Hartvigsen G, Basu A, Abbott P. Unintended Consequences of Tele Health and Approaches for their Solutions. Yearb Med Inform 2016;(1):41-6.

36. Veinot TC, Mitchell H, Ancker JS. Good intentions are not enough: how informatics interventions can worsen inequality. J Am Med Inform Assoc 2018:25:1080-8.

37. Ohno-Machado L. Understanding and mitigating the digital divide in health care. JAm Med Inform Assoc 2017;24(5):881.

38. McDonald CJ, Overhage JM, Mamlin BW, Dexter PD, Tierney WM. Physicians, information technology, and health care systems: a journey, not a destination. J Am Med Inform Assoc 2004:11:121-4.

39. Friedman C, Rubin J, Brown J, Buntin M, Corn M, Etheredge $\mathrm{L}$, et al. Toward a science of learning systems: a research agenda for the high-functioning Learning Health System. J Am Med Inform Assoc 2015;22:43-50.

40. Epstein RM, Fiscella K, Lesser CS, Stange KC. Why the nation needs a policy push on patient-centered health care. Health Aff(Millwood) 2010 Aug 1;29(8):1489-95.

\author{
Correspondence to: \\ Craig Kuziemsky \\ Telfer School of Management \\ University of Ottawa \\ Ottawa, ON \\ Canada \\ Tel: + 16135625800 ext 4792 \\ E-mail: Kuziemsky@telfer.uottawa.ca
}

